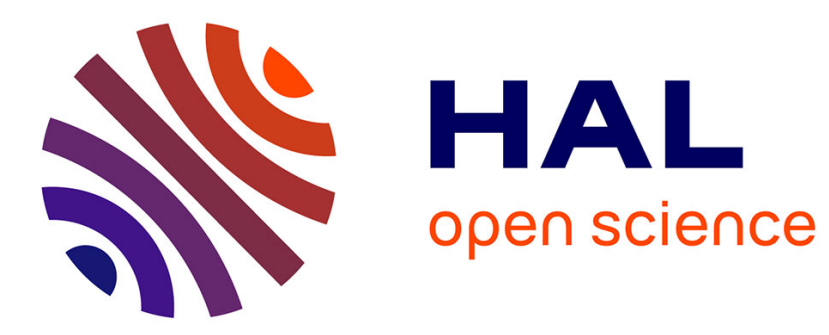

\title{
L'inauguration du musée d'art moderne et d'art contemporain de Nice en 1990
}

Philippe Poirrier

\section{To cite this version:}

Philippe Poirrier. L'inauguration du musée d'art moderne et d'art contemporain de Nice en 1990.

Vingtième siècle. Revue d'histoire, 1993, 38 (1), pp.62-73. hal-01545706

\section{HAL Id: hal-01545706 \\ https://u-bourgogne.hal.science/hal-01545706}

Submitted on 23 Jun 2017

HAL is a multi-disciplinary open access archive for the deposit and dissemination of scientific research documents, whether they are published or not. The documents may come from teaching and research institutions in France or abroad, or from public or private research centers.
L'archive ouverte pluridisciplinaire HAL, est destinée au dépôt et à la diffusion de documents scientifiques de niveau recherche, publiés ou non, émanant des établissements d'enseignement et de recherche français ou étrangers, des laboratoires publics ou privés. 


\title{
L'inauguration du musée d'art moderne et d'art contemporain de
} Nice en 1990

\section{Philippe Poirrier}

\begin{abstract}
The inauguration of the Nice Museum of Modem and Conteporary Art in 1990, Philippe Poirrier.

On 8 April 1990, the Franco-American sculptor Annan refused to inaugurate the retrospective of his work at the new Modem and Contemporary Art Museum of Nice in company of Jacques Médecin, mayor of Nice. The reason was the mayor's complecency vis-à-vis the National Front's theses. The incident originated a local and then a national affair that in which the artists, the elected officials of the Côte d'Azur and the minister of Culture, Jack Lang, were involved. The debate clearly illustrates some of the political stakes involved in cultural policies in France.
\end{abstract}

\section{Citer ce document / Cite this document :}

Poirrier Philippe. L'inauguration du musée d'art moderne et d'art contemporain de Nice en 1990. In: Vingtième Siècle, revue d'histoire, ${ }^{\circ} 38$, avril-juin 1993. pp. 62-73;

http://www.persee.fr/doc/xxs_0294-1759_1993_num_38_1_2680

Document généré le 05/05/2016 


\title{
L'INAUGURATION DU MUSÉE D'ART MODERNE ET D'ART CONTEMPORAIN DE NICE EN 1990
}

\author{
Philippe Poirrier
}

Voici, avec la montée du Front national et l'affaire de Carpentras en toile de fond, la culture niçoise dans tous ses états, prise en tenaille entre le prince et l'édile, à l'occasion d'une polémique précédant l'ouverture d'un musée. Nos politiques culturelles contemporaines sont, décidément, pleinement politiques. "Que dois-je faire?, a conclu le peintre Ben en juin 1990: dire que je ne supporte plus la désinformation et que je n'aime pas être manipulé, ni par les médias, ni par les Jack ou les Jacques ».

"A près la réception royale faite à A Jean-Marie Le Pen et à l'ancien Waf1 fen Ss Schoenhuber et les déclarations assez antisémites de M. Médecin, je n'ai pas eu le cœur d'inaugurer cette exposition main dans la main, sourire dans le sourire avec le maire de Nice." Cette déclaration, le 8 avril 1990, à l'Agence France-Presse du sculpteur francoaméricain Arman, annonce l'annulation de la rétrospective de l'artiste prévue pour l'inauguration, fin juin, du Musée d'art moderne et d'art contemporain de Nice

1 Le 7 avril, Arman avait fait parvenir aux rédactions de la presse française, par l'intermédiaire de la galerie Beaubourg, un premier texte manuscrit explicitant sa démarche. Resté confidentiel, seul Sud-Ouest le publie dans son édition du 8 avril, ce
(MAMAC) ${ }^{1}$. Ce refus ouvre alors un vaste débat auquel participent les principaux acteurs d'une politique culturelle: le prince, Jack Lang, l'édile, Jacques Médecin, et les milieux culturels parisiens et niçois. L'étude, sur trois mois, de cette polémique permet de mieux comprendre la place qu'occupe, à la fin des années 1980, la "culture" dans le discours des élus. Elle renseigne sur le rôle, singulièrement français, du ministère de la Culture et de celui qui matérialise et symbolise l'action culturelle de l'État : le ministre. De surcroît, cette approche met en évidence l'évolution du système politico-administratif depuis les lois de décentralisation en 1982. Enfin, l'analyse de la perception, ou plutôt des perceptions, d'un *boycottage " qui, par ses répercussions dans l'espace public, donne sens à un geste individuel, apporte des éléments sur la construction et la diffusion d'un "événement " dans une société désormais fortement marquée par l'omniprésence de la médiatisation ${ }^{2}$.

texte ne permit pas la diffusion du refus d'Arman. C'est donc la version de l'AFP, plus précise, et reprise par l'ensemble des médias, qu'il faut retenir.

2. Nous tenons a remercier ici la direction du MAMAC et Isabelle Goetzmann, responsable du service de documentation du musée. 
O ÉPHÉMÉRIDE

\section{Le refus de l'artiste}

Alors que le 2 avril 1990 l'AFP plaçait l'inauguration du MAMAC sous le signe de la première rétrospective en France d'Arman, le désistement de l'artiste, diffusé le 8 , semble surprendre, même si les prises de parole civiques du plasticien sont déjà nombreuses. En effet, les convictions éthiques d'Arman ne sont pas nouvelles: membre du Mouvement contre le racisme et pour l'amitié entre les peuples (MRAP), ancien président, aux États-Unis, de l'Association des artistes contre l'apartheid, le plasticien s'était déjà élevé en 1974 contre le jumelage entre la ville de Nice et Le Cap. Cette décision émane d'un sculpteur qui bénéficie d'une grande notoriété, tant au plan local - il apparaît en septième position dans le palmarès culturel, établi par des journalistes niçois, "les dix qui comptent ", et publié par Télérama ( $\mathrm{n}^{\circ}$ 2033, décembre 1989), devancé respectivement par Jacques Weber, JeanMarie le Clézio, Pierre Médecin (frère du maire et directeur de l'Opéra), Claude Fournet, Ben et Hélène Jourdin-Gassin, galeriste et présidente d'Art jonction internationale-qu'au plan international: signataire le 27 avril 1960 de la *Déclaration constitutive " des Nouveaux Réalistes, proche ami de Klein et niçois comme lui, Arman est, avec Vasarely, l'artiste français qui bénéficie à la fin des années 1970 de la notoriété la plus forte auprès des instances de légitimation du monde de l'art ${ }^{1}$.

Son refus n'est pas la seule initiative de protestation prise contre l'attitude politique du maire de Nice. Dès le 7 avril, le peintre et dessinateur niçois Baudoin

\footnotetext{
1. Nous suivons ici les - courbes d'estime - élaborées par Annie Verger, - L'art d'estimer l'art. Comment classer l'incomparable ', Actes de la recherche en sciences soctales, mars 1987, p. 105-121. Un classement international publié en 1983 par la revue Capital confirme ce point : Arman est $23^{c}$, devancé par Yves Klein (12), Daniel Buren (19), mais devant Martial Raysse (49).
}

exprime ouvertement son désaccord en placardant, place Garibaldi, à deux pas du nouveau musée, des affiches sur lesquelles on peut lire: "Je suis Niçois et j'aime ma ville mais j'ai honte de mon maire. 2 . La réaction de la ville de Nice est alors modérée et André Barthe, délégué aux Affaires culturelles et aux beaux-arts, se contente d'exprimer les regrets de la municipalité 3 .

Dès le 9 avril, Jack Lang envoie à Arman un télégramme de félicitation. Le lendemain, un communiqué du ministère de la Culture signale le geste du ministre et ajoute: "Au moment où Monsieur Médecin récidive et revendique à 99,9\% les thèses du Front national, Jack Lang espère que d'autres artistes accepteront de suivre l'exemple d'Arman * et qu'*audelà du cas particulier de M. Médecin, on peut souhaiter que puisse s'organiser bientôt un mouvement de boycott des artistes et des intellectuels à l'égard de tous les hommes politiques qui pactisent avec l'antisémitisme " 4 . La réaction de Jack Lang, au-delà d'un geste de citoyen, par son mode de médiation, apparaît plus comme une position officielle du ministre de la Culture. Elle infléchit nettement le refus d'Arman et donne une implication politique à l'événement.

Le vou de Jack Lang est exaucé quelques jours plus tard. Dans sa livraison du 26 avril, L'Événement du jeudi approuve le refus d'Arman et souligne, avant de donner la parole à dix artistes, l'étonnante solidarité d'un milieu de l'art réputé concurrent . Si la condamnation des propos de Jacques Médecin fait l'unanimité,

2. L'affiche est publiée par Var-Matn le 8 avril.

3. Nice-Matin le 10 avril et Present le 25 avril. Des représentants de la municipalité niçoise prendront contact avec Arman à New York pour qu'il infléchisse sa décision. Le sculpteur accepta à condition que Jacques Médecin fasse des excuses publiques aux trois conseillers municipaux démissionnaires et qu'il les réintègre dans son conseil $\cdot$ afin que la communauté juive retrouve sa dignité. Le sculpteur ne fut pas entendu. (Voir l'entretien publié par La Vie, le 21 juin 1990.)

4. Texte publié par Libération le 13 avril. 
les modalités de la réponse restent sujet à discussion et divisent ses artistes, proches, pour la plupart, de l'École de Nice ou dans son sillage: Jacques Monory, Daniel Buren, Ben, Sacha Sosno, Bernard Pagès, Hervé Di Rosa, Dolla, Daniel Deseuze, Pierre Soulages et Daniel Spoerri. Le même jour, Patriote-Côte d'Azur publie, dans le même esprit, les réactions individuelles d'artistes et critiques d'art niçois ou liés à la ville : Michel Butor, Bernard Pages le seul artiste avec le peintre Thupinier à n'avoir pas accepté de serrer la main de Jacques Médecin, en 1985, à l'ouverture de l'Acropolis -, Louis Chacalis, Luc Warneck, Charvolen, Henri Maccheroni, Lola Gassin, Armand Sholtes et le critiquethéoricien des Nouveaux Réalistes, Pierre Restany. Ces prises de position individuelles cèdent bientôt la place à différentes déclarations de principe collectives.

Une seconde réaction, sous la forme d'un manifeste, est ainsi publiée par Globe, mensuel de sensibilité proche de la Rue-de-Valois, le 2 mai. Cet appel, "L'Art contre l'infamie , est signé par treize artistes internationaux reconnus à la fois par la critique, le marché de l'art et, pour certains, par la commande publique : Arman, Jean Bazaine, Daniel Buren, Pierre Klossowski, Roy Lichtenstein, Alfred Manessier, Édouard Pignon, Jullian Schnabel, Pierre Soulages, Daniel Spoerri, Franck Stella, Jean Tinguely et Zao Wou Ki ${ }^{1}$ :

- Bouleversés par les récentes déclarations du maire de Nice, treize artistes internationaux ont décidé de protester contre ce qui leur apparaît une infamie. À ce titre, ils déclarent solennellement refuter toute invitation à exposer dans les lieux relevant de cette municipalité. Comme le gouvernement des hommes, l'art dans la cité est pour eux affaire de morale. Universel par destination, il ne saurait venir cautionner quelque acte public porteur d'ostracisme. Toute atteinte à l'honneur des hommes, pour des raisons d'appartenance, est une atteinte à la démocratie et, par là, fait injure aussi à l'art comme bien commun à tous.

1. L'AFP l'annonce dans une dépêche du 30 avril.
Face à la gangrène antisémite et raciste qui cherche ici ou là à se frayer droit de cité, les soussignés s'engagent à appliquer le boycott par l'art, au-delà de Nice, à tous les hommes et institutions politiques qui se montreraient complices de l'infamie .

Ce manifeste, qui élargit la perspective, dépasse la conjoncture niçoise et lui donne un net caractère international, est accompagné d'un court texte d'Arman - nous sommes tous des racistes "-, défense d'un monde pluri-culturel et apologie du droit à la différence. Pour sa part, le milieu culturel niçois ne reste pas inactif et une centaine d'artistes signent un texte par lequel ils condamnent * résolument et définitivement • les propos de Jacques Médecin et son allégeance *de plus en plus manifeste - au programme du Front national ${ }^{2}$.

Au début de mai, la section française de l'Association internationale des critiques d'art (AICA) s'associe à son tour au boycottage. Les médiateurs, personnages clefs du monde de l'art contemporain, rejoignent ici les créateurs et diffusent le communiqué suivant ${ }^{3}$ :

- Pour la première fois, un grand nombre de journalistes et de critiques d'art en France a décidé de boycotter l'inauguration d'un nouveau musée. La profession est bien entendu favorable à toute initiative concernant l'art et les artistes. Toutefois, elle estime que les prises de position antisémistes et racistes de Jacques Médecin, maire de Nice et autorité de tutelle du nouveau musée d'art moderne et d'art contemporain, l'empêchent de rendre compte de cet événement. Les conséquences de toute attitude d'exclusion ont été suffisamment monstrueuses dans l'histoire pour que la critique d'art marque dès maintenant son refus de cautionner une entreprise culturelle placée sous de tels auspices. Les critiques d'art approuvent le refus d'Arman d'y présenter sa première grande rétrospective en France ainsi que l'action de tous les artistes internationaux qui ont exprimé leur indignation. Ils tiennent à alerter l'opinion: l'art ne saurait en aucun cas être associé à l'intolérance, au racisme .

2. Publié par le Patriote - Cốte d'Azur le 4 mai 1990.

3. AFP, le 9 mai 1990. 


\section{Le boycottage de l'État}

Le relais est alors repris par l'État et donne à la polémique une dimension nouvelle. Au cours d'une conférence de presse dans les salons de l'aéroport de Nice, Jack Lang, le 9 juin, indique qu'à la suite de demandes d'artistes l'État ne prêtera pas certaines ouvres des collections publiques au musée. Le ministre ajoute qu'il ne se rendra pas, comme il était prévu, à l'inauguration. Ce prêt, d'une dizaine d'œuvres de Jasper Johns, Larry Rivers, Martial Raysse, Spoerri, Niki de Saint-Phalle et Tinguely, provenant des collections du Centre Beaubourg et du Fonds national d'art contemporain, devait combler certaines lacunes du musée. En fait, la décision de Jack Lang officialise et rend publique une initiative annoncée le 9 mai à la direction des musées de Nice par Jean-Hubert Martin, directeur du Musée national d'art moderne (Centre Georges Pompidou) et bientôt suivie, non sans quelques réticences préalables, d'une démarche analogue de François Barré, responsable du FNAC.

Malgré ces péripéties, l'inauguration du MAMAC a lieu le 21 juin en présence d'environ deux mille personnes: NiceMatin parle même d' "une levée en masse . Cette mobilisation doit sans doute beaucoup à l'appel de l'Association des amis du maire, un des rouages essentiels du "médecinisme " et soutien inconditionnel au * patron*1. Jacques Médecin, aux côtés de Jean-Claude Gaudin (UDF), président du Conseil régional PACA, entouré de Yvon Briant, président du CNI - "s'évadant quelques heures de Paris ", note France indépendante-et de nombreux parlementaires de la région ${ }^{2}$, sait

1. Sur le rôle des associations paramunicipales et le système de clientèle niçois, of. Pierre Tafani, - La tyrannie a Nice au temps de Jacques Médecin ou des mille et un effets de la décentralisation -, Hérodote, 62, 1991, p. 115-124.

2. Suzanne Sauvaigo, Martine Daugreilh, Charles Ehrmann, Christian Estrosi, Pierre Merti, Rudy Salles, députés des AlpesMaritimes; Charles Ginelly, Pierre Laffitte, Honoré Bailet, José Balarello, sénateurs. faire de cette manifestation une victoire personnelle. Plusieurs artistes, refusant le boycottage, sont également présents : Sosno, Fahri, Setman, J.-P. Ozenda, Tréal, Nigel Ritchie, France Raysse, Jarry, Max Cartier. Parmi ces quelques créateurs, la présence de Ben, affublé d'une vesteécriteau sur laquelle on peut lire « je suis contre le racisme et contre le diktat de Paris n, traduit bien la diversité des attitudes.

Le lendemain, une centaine d'artistes menés par Ben et le sculpteur Angel, refusant explicitement la notion de "contre-inauguration", effectuent une "simple visite dans un lieu que nous pensons important pour l'activité artistique et culturelle. 3 . Non sans ironie, Jean Mas propose également une inauguration-performance pour sa "visite inaugurale" le 29 juin 4 . Enfin, le 19 juillet suivant, Ben fait visiter les nouvelles cimaises niçoises à UltraViolet (alias Isabelle Dufresnes), l'ancienne compagne du "pape. new-yorkais du popart Andy Warhol. Après les politiques, les créateurs s'approprient le lieu: le musée peut ouvrir.

Ces nombreuses prises de position, des politiques comme des artistes, masquent le silence d'un troisième acteur: le conservateur-commissaire. Claude Fournet, directeur des Musées de la cité et appelé à Nice en 1975 pour y créer un musée d'art moderne, demeure volontairement en retrait et se cantonne dans son rôle professionnel: « Mon point de vue, je l'exprime à travers le MAMAC que j'ai contribué à créer... Lorsqu'on voudra me dicter mes choix, je prendrai mes responsabilités", déclarera-t-il en février $1992^{5}$. Pierre Chaigneau et Gilbert Perlein, conservateurs du MAMAC et commissaires de la rétrospective Arman - en ce sens, la res-

\footnotetext{
3. Voir le compte rendu dans Nice-Matin du 23 juin 1990.

4. La pétition de principe de Jean Mas est publiée par Artbèmes, juillet-août 1990

5. Le Monde, 29 février 1992. C'est néanmoins Claude Fournet, bon connaisseur du - médecinisme , qui recommanda a Jacques Sallois, directeur des Musées de France, d'être absent pour l'inauguration.
} 
ponsabilité de l'exposition leur revient, ce qui discrédite un des arguments avancés par Jacques Médecin pour expliquer le refus d'Arman: le retard du sculpteur dans la préparation-, respectent le droit de réserve exigé des fonctionnaires et restent cantonnés dans la présentation de la collection et la défense des choix muséographiques.

\section{O PERCEPTIONS}

Pour apprécier le geste d'Arman, il convient de prendre la mesure de la médiatisation du débat qui s'en suivit, jugée excessive par Le Monde dans sa livraison du 20 juin. Une première pesée quantitative permet d'évaluer la montée en puissance d'une polémique bien relayée par la presse ${ }^{1}$ : d'avril à octobre 1990 , près de trois cent cinquante périodiques couvrent la polémique, devenue un * événement ". Cette médiatisation suit alors le développement de l'affaire. Des trente-deux articles qui font suite au désistement d'Arman - essentiellement dans la presse régionale française qui reprend une dépêche de l'AFP -, la montée en puissance est nette dès qu'interviennent les différentes protestations collectives: plus de cent articles de la fin d'avril jusqu'au boycottage de l'État au début de juin. L'intervention de Jack Lang a permis de passer à un stade supérieur: deux cents articles précédent ou suivent l'inauguration. Mais surtout, la presse internationale, avec environ cent trente articles, notamment en Allemagne et aux États-Unis, se fait l'écho de l'intervention ministérielle. Les principaux titres des métropoles étrangères soulignent, en personnalisant le débat, cet affrontement entre Jacques Médecin et Jack Lang: The European le 22 juin, La Stampa le 18, El Pais le 28 juillet, le Herald Tribune du 14 juin, Die

\footnotetext{
1. Le corpus est ici constitué des articles recensés par la revue de presse du musée. S'il n'est pas exhaustif, il permet pour le moins de décrire un trend.
}

Welt le 22 juin, L'Observer le 20, Der Spiegel le 2 juin ou encore le New York Times le 13. Une analyse qualitative permet d'affiner la perspective.

\section{Un geste moral}

L'antiracisme qui justifie le refus d'Arman place d'emblée le débat sur le terrain de la morale. Le sculpteur revendique pleinement sa qualité d'intellectuel : - Il s'agit avant tout d'une réaction personnelle. Jouissant d'une certaine notoriété, je me dois de l'employer pour les idées que je défends. Et je suis farouchement contre le racisme, l'intolérance, l'antisémitisme. 2 . Cette nécessaire intervention de l'artiste dans les affaires de la cité légitime les différentes prises de position collective sous la forme de manifestes. Dans cette perspective, Jack Lang souligne combien le ministre de la Culture se doit, par un geste * symbolique *, de respecter ce * droit moral - des artistes. Ces prises de position s'inscrivent dans un mouvement amorcé à l'occasion des élections partielles de Dreux en 1983: Jean-François Sirinelli a bien montré combien le combat *antiraciste - permet alors une passerelle entre les socialistes et les intellectuels, car la cléricature de gauche, muette depuis 1981, y avait trouvé un moyen de revitaliser ses idéaux ${ }^{3}$.

Quant à Jack Lang, son combat contre le racisme ne s'est pas démenti depuis plusieurs années. En 1983, avec Georgina Dufoix, secrétaire d'État chargé de l'Immigration, il a soutenu la première marche antiraciste. En juin 1985, le ministre de la Culture a apporté son soutien financier et logistique-Christian Dupavillon fut le coordinateur des opérations - au concert organisé, place de la Condorde, par SOS-

2. La Vie, le 21 juin 1990. Rappelons la définition proposée par Pascal Ory: - I'intellectuel est un homme du culturel mis en situation d'homme du politique - (Pascal Ory (dir.), Dernières questions aux intellectuels, Paris, O. Orban, 1990, p. 24).

3. Jean-François Sirinelli, Intellectuels et passions francaises, Paris, Fayard, 1990, p. 310-318. 
Racisme. Jack Lang a joué également le rôle de médiateur lorsqu'il s'agit de trouver des mécènes pour la jeune association et Pierre Bergé, P-DG d'Yves SaintLaurent, déjà financier du mensuel Globe, devint alors un des principaux bailleurs de fonds de l'organisation animée par Harlem Désir. À l'automne 1988, Jack Lang recommandait Pierre Bergé à François Mitterrand pour être nommé à la tête des Opéras de Paris. En mai 1989, Harlem Désir est venu promouvoir à Blois un festival pluri-culturel organisé par le nouveau maire, Jack Lang. Enfin, lors de l'affaire des "foulards islamiques", à l'automne 1989, le ministre de la Culture a défendu SOS-Racisme au moment où l'alternative entre intégration et droit à la différence déchirait une partie des socialistes ${ }^{1}$.

Cette mise en avant du droit moral de l'artiste à récuser le racisme est bien reçue par la majorité des revues spécialisées de la capitale: l'éditorial de Beaux-Arts en juin souligne "le geste inaugural " d'Arman; $A r t$ Press (juin), Opus international (juilletaoût), Ancrages Magazine (octobre) ou encore Arts Magazine (juillet-août), qui devaient consacrer des articles à l'ouverture du musée, publient à leur place le communiqué de l'AICA; Galerie Magazine (mai) en publie la version bilingue et met en avant le sacrifice d'Arman qui "mérite respect et admiration. L'unanimité règne aussi dans une grande partie des mensuels et des hebdomadaires (Globe, Actuel, L'Express, Le Point, L'Événement du jeudi, Politis, Le Nouvel Observateur) et dans la presse communiste (Rouge, Révolution, L'Humanitê).

Jacques Médecin, lui, a aussitôt récusé la bonne foi du sculpteur francoaméricain et indiqué que celui-ci n'était pas prêt. Son refus masquerait de simples

1. Richard Desneux, Jack Lang. La culture en mouvement, Paris, Favre, 1990, p. 172, et Mark Hunter, Les jours les plus Lang, Paris, Odile Jacob, 1990, p. 228-231, 286-287 et 302-303. mobiles mercantiles: son marchand, Pierre Nahon, de la Galerie Beaubourg, ayant acheté une boutique en face du musée, le sculpteur préférerait réaliser des affaires avec cette galerie ${ }^{2}$. Le Figaro, le lendemain de l'inauguration, rappelle que le maire de Nice a ensuite admis qu'il avait prononcé une a parole malheureuse . Mais surtout François Terré reprend, sur le terrain juridique, la notion de droit moral destinée, souligne-t-il, avant tout à protéger la personnalité de l'artiste. À partir de la jurisprudence, il note que si le propriétaire d'un objet d'art ne peut le mutiler (voir l'affaire Bernard Buffet), il peut le détruire (ce fut jugé dans l'affaire du curé de Juvisy). Ainsi, le propriétaire peut disposer d'une ouvre d'art comme il l'entend, sauf abus : « Rien de tel, a priori, s'agissant de l'exposition niçoise. ". Le quotidien du groupe Hersant insiste davantage sur le sens donné par Jacques Médecin à l'inauguration.

\section{Un geste jacobin}

L'absence du ministre de la Culture, du directeur des Musées de France, Jacques Sallois, ancien directeur du cabinet de Jack Lang, et de la presse nationale, ainsi que le relatif échec du boycottage ${ }^{3}$, permettent au maire de Nice de dénoncer à la fois le poids du ministère de la Culture et "le terrorisme intellectuel " des médias de la capitale. A l'emplacement que devraient occuper les œuvres du MNAM et du FNAC, le maire a fait placer un carton vengeur: * Ici doit être présentée une œuvre appartenant au patrimoine national. Elle est retenue arbitrairement à Paris *. Se plaçant sous le signe du *diktat * parisien, Jacques Médecin se pose donc

2. Le Quotidien de Paris, 5 mai 1990 ; interprétation reprise dans Jacques Médecin, Et moi je vous dit ... ma vérité. Entretien avec Henri-Christian Giraud, Paris, M. Lafond, 1991, p. 268.

3. L'Action Nice-Côte d'Azur, périodique contrôlé par la municipalité et animé par l'écrivain Raoul Mille, ne manque pas de publier, dans sa livraison du mois d'août, un cliché montrant les artistes (Arman, César, Sosno ...) visitant le chantier du musée aux côtés de Jacques Médecin pendant l'été 1988. 
en fervent défenseur de la décentralisation culturelle face au a jacobinisme centralisateur * du ministère de la Culture. Il met en avant les modalités de financement du MAMAC : pour un coût total avoisinant les deux cents millions de francs, le musée a été financé à $65 \%$ par la ville de Nice, $10 \%$ par le département, $8 \%$ par la région et $17 \%$ seulement par l'État, la participation de ce dernier n'ayant été obtenu qu'en 1987, sous le ministère de François Léotard. Le musée, depuis 1986, bénéficie en outre d'un budget d'acquisition de plus six millions par an: cinq attribués par la ville, avec complément de l'État pour un million et du FRAM pour un demi-million. Le coût du fonctionnement annuel, d'après le budget prévisionnel, est évalué à dix millions. Avec le nouveau Théâtre (163 millions de francs), ce double chantier culturel, conçu par l'architecte Yves Bayard, est alors le plus important après celui de l'Opéra-Bastille. De plus, les $15 \%$ du budget municipal consacrés en 1990 à la culture placent la municipalité de Nice parmi les villes affichant un très net volontarisme culturel et traduisent un effort amorcé au milieu des années 1970: 7,6\% en $1978,8,7 \%$ en $1981,11,1 \%$ en 1984 et $14,5 \%$ en 19871 .

Quant au soutien de la région Provence-Alpes-Côte d'Azur, il s'inscrit dans le cadre d'une politique culturelle qui vise notamment à doter le territoire régional d'équipements à vocation européenne: outre le MAMAC, le Musée d'art antique et la médiathèque d'Arles, le théâtre de Cap, la Fondation Templon à Fréjus, le FRAC à Marseille ou encore la bibliothèque Méjane à Aix-en-Provence. En plus des quelque vingt millions de francs

1. En 1987, ces chiffres placent Nice juste derrière Strasbourg $(23,7 \%)$, Lyon $(21,7 \%)$, Bordeaux (15,7\%), Lille (15,7\%), Grenoble $(15,6 \%)$ et Brest $(15 \%)$. Si on retient le ratio francs/habitant, somme toute plus significatif, Nice se situe, avec 1381 F./hab., en troisième position derrière Bordeaux (1 664 F.) et Grenoble (1 463 F.). Voir Les dépenses culturelles des communes. Analyse et évolution 1978-1987, Paris, DEP/La Documentation française, 1991, p. 218-219. consacrés à l'investissement dans le domaine des arts plastiques depuis 1987, la région participe à des actions plus ponctuelles, à hauteur de vingt millions de francs également: la Fondation Van Gogh à Arles, le symposium de la sculpture de Digne mais aussi la biennale d'Art contemporain de Brignolles 2.

Au-delà de ces considérations strictement culturelles, l'édification du MAMAC et l'achèvement de la Promenade des arts (l'Acropolis - Palais des congrès, le Théâtre et le Musée) sont explicitement présentés par la municipalité de Nice comme des symboles de l'alliance du dynamisme économique et de la vocation culturelle internationale de la ville. Le traitement urbanistique retenu, qui fait de la Promenade des arts le point de jonction entre la ville ancienne et la ville neuve, se veut la matérialisation architecturale d'une modernité respectueuse de la tradition ${ }^{3}$. Ainsi, et à l'image des principales métropoles françaises, le paradigme qui légitime l'intervention des édiles dans le champ culturel ressortit désormais des stratégies de communication ${ }^{4}$ et de concurrence urbaine 5 .

Le boycottage des institutions publiques suscite en outre, dès le début de juin, une vague de protestations des élus de la région niçoise. Pierre Laffitte, sénateur des Alpes-Maritimes et vice-président de

2. Si l'aide de l'Etat pour la construction du MAMAC peut sembler modeste, il faut cependant souligner que, depuis plusieurs années, la Région PACA bénéficie pour ses musées de crédits d'État en nette augmentation : 7,7 MF en 1987, $13 \mathrm{MF}$ en $1988,24,7 \mathrm{MF}$ en 1989 et $27,1 \mathrm{MF}$ en 1990 . Fn 1989 , la Région reçoit ainsi prés de $33 \%$ des 75,5 MF de crédits de l'État pour les musées, devant la Corse $(22 \%)$, Rhône-Alpes $(13,7 \%)$, la Lorraine $(8,7 \%)$, la Bourgogne $(7 \%)$. Ces chiffres sont cités par Patrice Conodera, - L'Etat et les musées en Provence-Alpes-Côte d'Azur :, Le Musée en mouvement, Toulon, ADAC-Conseil général du Var, 1990, p. 25-33.

3. La lettre du maire, mai-juin 1990.

4. Le budget de communication pour le lancement du Musée es de 700000 francs d'après le neustetter Stratégies du 30 avril 1990.

5. Sur ce point, voir Mireille Pongy, - Politiques culturelles terntoriales : une approche en termes de référentiel , Les Papiers du GRESE, 6, 1989, p. 19-32. Signe de cette évolution : a l'automne 1991, la ville de Nantes lancera dans l'ensemble des médias nationaux une campagne axée sur cette complémentarité de l'économique et du culturel (- Et si une ville etait sûre que le bouillonnement culturel favorise l'effervescence industrielle? $\cdot$ ). 
la commission des Affaires culturelles du Sénat, dénonce le 11 juin la p politique culturelle sélective " du ministre de la Culture. Se plaçant sur le terrain juridique, il pose la question de l'utilisation du patrimoine public: "Tout artiste, à titre personnel, a le droit, comme le sculpteur Arman ou d'autres, de ne pas exposer dans ce musée. Vous-même (J. Lang), ou certains fonctionnaires, avez le droit de ne pas participer à son inauguration. Mais les gestionnaires d'œuvres qui appartiennent au patrimoine national n'ont pas le droit de refuser, pour des raisons personnalisées, le prêt d'œuvres à un organisme public " 1 . Sur ce même terrain, José Balarello, sénateur-maire de Tende, rappelle qu'en vertu de la règle du parallélisme en droit administratif, aucun arrêté n'ayant annulé les deux arrêtés du 2 août 1989 accordant les prêts et dépôts d'œuvres des musées nationaux, ces derniers restent en vigueur et doivent donc être appliqués sans délais. Dans le même esprit, Rudy Salles, député des Alpes-Maritimes, regrette que le gouvernement et le ministre de la Culture n'accordent pas aux Niçois les mêmes droits qu'aux autres citoyens dans le domaine culturel. Le sénateur Honoré Bailet, futur maire de la ville, regrette l'absence du ministre de la Culture * d'autant plus que le motif invoqué me paraît relever davantage de considérations politiques que de préoccupations prétendument morales et s'accompagne d'une rétention d'œuvres d'art appartenant au patrimoine national que vous vous étiez engagé à mettre à la disposition du public niçois, qu'ainsi vous prenez en otage $\times 2$. Le Parti communiste adopte, non sans nuance, une attitude voisine. Si la presse parisienne du parti soutient les artistes, ses instances locales, tout en reconnaissant aux plasticiens "toute liberté d'exhibition de leurs œuvres ", critiquent fermement,

1. AFP, 12 juin 1990.

2. Lettres publiées par Nice-Matin les 14,15 et 16 juin 1990. en revanche, "la décision de Jack Lang de refuser de prêter au musée de Médecin des toiles et sculptures émanant des musées nationaux", et Charles Caressa, secrétaire de la fédération locale du PCF, dénonce l'attitude ministérielle responsable d'un "déficit culturel pour nos citoyens ${ }^{3}$. Les communistes locaux décident d'ailleurs de soutenir la contre-inauguration des artistes niçois.

La décision de Jack Lang a placé ces derniers, il est vrai, dans une position inconfortable. Dès leur mise au point collective du 4 mai, les artistes niçois ont souligné que leur protestation n'impliquait pas un renoncement à leur activité culturelle locale et surtout ont refusé de faire tout amalgame entre les propos du maire et la fréquentation des lieux culturels gérés par la municipalité. La position de Ren, réitérée à maintes reprises, synthétise assez bien cette position. Tout en proclamant son indignation, il refuse de signer le manifeste "L'art contre l'infamie", dénonce la réaction orchestrée, pilotée par Jack Lang, et la réduction de Nice à une "ville pestiférée " 4 . La revue Arthèmes, de Nice, s'estime d'ailleurs dans l'obligation de publier, pour la première fois depuis sa création en 1983, un éditorial pour expliquer sa position. Sous le titre "Non au Racisme. Oui à la Création à Nice ", Marie-Lou Lamarque, sa directrice, rejoint Ben et s'indigne contre l'établissement * d'un nouveau racisme... aussi intolérant que le premier qui enferme la création niçoise dans un nouveau ghetto . Elle souligne combien la question initiale, clairement morale et éthique, s'est trouvée déformée par des "intérêts politiques *.

Cette attitude du milieu culturel niçois traduit un changement, matérialisé en 1985 à l'exposition "Autour de Nice. à l'Acropolis, véritable préfiguration du

3. L'Humanité, 20 juin 1990 et Révolution, 22 juin 1990.

4. Le Quotidien de Parix 10 mai 1990 , et ses nombreuses prises de position dans Artbèmes en mai et juin. 
futur musée ${ }^{1}$, par rapport à la méfiance qui prévalait auparavant au sujet de la politique culturelle municipale. En 1978, les acteurs culturels locaux notaient : "C'est donc sur le plan du rapport de force que se situe la culture, et la liste serait longue des qualificatifs d'incompétences, de lacunes, de volonté délibérée d'asservir ou d'utiliser, dont il faudrait accabler son adversaire: le pouvoir " 2 . Ce changement d'attitude doit sans doute beaucoup à la politique engagée à partir de 1977 par trois acteurs clés de la société culturelle niçoise: André Barthes, adjoint au maire chargé des Beaux-Arts, Lucien Pampaloni, directeur du service des Affaires culturelles et surtout Claude Fournet. La multiplication d'expositions d'art contemporain à la galerie municipale des Ponchettes et à la galerie de la Marine transformée en Galerie d'art contemporain, tout en sensibilisant le public local, avait permis aussi de "réconcilier " les artistes locaux et la municipalité 3 .

Sans doute conscient des avantages politiques que Jacques Médecin pouvait retirer d'une telle interprétation de la polémique, Jack Lang, le 18 juin, à trois jours de l'inauguration, précisait donc qu'il avait suggéré aux artistes d'interrompre le boycottage après le 21 juin et ajoutait qu'il fallait distinguer la ville de son maire.

1. En fait, essentiellement les écoles de Nice, Support-Surface, certains Nouveaux Réalistes comme Villeri, Viallat, Rousse, Romeda, Poirier, Pagès, Le Gac, Klein, Hains, Gainon, Moya, Fahri, Dezeuze, Chubac, Charloven, Ben. La collection du MAMAC sera complétée par l'acquisition et la constitution, à partir de 1985, d'œuvres du pop ant américain, mouvement contemporain des Nouveaux Réalistes, du Color Field Painting et des tendances intemationales, marquées dans les années 1980 par le retour a la figuration. Pour cette politique d'acquisition, Claude Foumet bénéficia en 1987-1988 de l'effondrement de la Bourse de New York et des incertitudes causées par les nouvelles lois fiscales américaines en matière de donation aux musées.

2. Culture dans la ville: sttuation a Nice. Lture blanc, Nice, Informations culturelles, 1978, p. 3.

3. Claude Fournet, - Les musées de Nice ", Kanal, 5, 1984, p. 10-13, et - Les raisons d'un Musée, Connaissance des Arts. Spécial musées de Nice, 1990, p. 10-19.

\section{Un geste politique}

C'est néanmoins l'observation du contexte politique qui donne les meilleurs éléments nécessaires à la compréhension du débat. La réception de Jean-Marie Le Pen à la mairie de Nice le 30 mars, la démission des conseillers municipaux israélites à la suite du vote du budget municipal par les élus du Front national, la "phrase malheureuse de Jacques Médecin devant les micros d'Antenne 2 le 2 avril - Je ne connais pas un Israélite qui refuse un cadeau qu'on lui offre, même si le cadeau ne lui plaît pas. Je ne connais pas non plus un maire qui refuse les voix qui lui sont apportées * - s'inscrivent dans une conjoncture où le racisme et l'antisémitisme font depuis plusieurs semaines la une des journaux. Le monde politique, alerté par la montée du Front national et du racisme quotidien, tente de prendre la mesure du phénomène: à preuve, le Rapport au Premier ministre sur la lutte contre le racisme et la xénophobie, la Plan national de lutte contre le racisme le 27 mars, la table ronde à Matignon sur le racisme du 2 avril et l'adoption le 2 mai, à l'initiative du Parti communiste, d'une loi destinée à renforcer la répression des actes racistes, antisémites et xénophobes. De plus, le $10 \mathrm{mai}$, la connaissance de la profanation du cimetière juif de Carpentras a déclenché une indignation unanime du monde politique. Le Front national, jugé moralement responsable de cet acte, a été mis au ban de la nation et, du 10 au 18 mai, les manifestations consensuelles contre l'extrême droite ont été nombreuses ${ }^{4}$. Dans cette conjoncture, les propos de Jacques Médecin, pourtant familier des outrances verbales, ont pris inévitablement un relief singulier. Le 4 avril, Jack Lang demande solennellement à Jacques Chirac et Valéry Giscard d'Estaing de dénoncer clairement

4. Sur ce contexte, voir Nonna Mayer, - L'affaire Carpentras -, L'Histoire, 148, 1991, p. 10-14. 
et fermement l'attitude du maire de Nice. Dans ce contexte, la profanation de Capentras et ses suites ont donc pu jouer un rôle d'accélérateur du boycottage du musée. Et dans sa livraison de juillet-août, Globe n'a pas craint d'associer les deux affaires de Carpentras et Nice: *L'art contre la barbarie ", titre-t-il, sous la rubrique "Résistances".

Dès le début de juin, Jacques Médecin dénonce donc le "diktat" et accuse Paris de vouloir punir les Niçois qui ont mal voté. La presse d'extrême droite place d'emblée l'affaire sur le terrain politique. Le journal du Front national, National Hebdo, se fait l'écho, sous la rubrique - Dictature , des propos de Jacques Médecin contre l'attitude de Jack Lang, désormais symbole de :l'arbitraire et du fait du prince" 1 . Dans son numéro du 29 juin, Rivarol voit dans le boycottage " les ravages idéologiques du pouvoir culturel abandonné à la gauche * et exprime ses inquiétudes sur la maintien d'un Etat de droit. Présent indique que c'est un * procès stalinien " qui est fait à Jacques Médecin et reprend comme titre un des propos tenus par ce dernier lors de l'inauguration: "Les deux seuls conservatoires du marxisme: la France et l'Albanie*. Le ministre de la Culture, * éructant ses grotesques anathèmes du haut de ses mini-colonnes de Buren", demeure la principale cible ${ }^{2}$. A l'opposé, le Parti communiste souligne que cette "sanction politique "n'est sans doute pas la meilleure arme à opposer aux * idées fascisantes" du maire de Nice. Le débat d'idées s'enlise alors en joutes verbales et voit s'affronter anticommunisme et antifascisme.

Cette polémique, initialement liée à des considérations éthiques, apparaît à certains comme un combat politique visant essentiellement à contrer Jacques Méde-

1. Nattonal Hebdo, 5 juillet 1990.

2. Présent, 23 juin 1990. cin, allié du Front national. Certaines revues vont donc se refuser à faire l'amalgame. Ainsi Connaissance des arts, qui publie d'ailleurs un numéro spécial sur Nice, estime que le boycottage du musée est une erreur de jugement. Son éditorial de juillet-août se refuse à faire de l'œuvre d'art une sorte d'otage des problèmes politiques et souligne, non sans raison, que les revues spécialisées se sont ridiculisées en publiant parfois leurs appels à quelques pages seulement d'une publicité payante pour l'inauguration du musée 3 . Dans le même sens, Luc-Élysée Serraf, éditorialiste de La Cote des arts, fustige les "méfaits d'une politique politicienne qui partage, scinde, sépare et soupçonne. aux dépens de l'art, et appelle au refus de toute confusion entre la politique et l'art ${ }^{4}$. Et Valeurs actuelles (16-22 juillet) dénonce «le prétexte à des règlements de compte "qui portent ombrage à un événement culturel "qui méritait plus de dignité . Ce poids du politique masque, dans une large mesure, le débat sur la fonctionnalité du musée et sur l'opportunité et la qualité esthétique des collections exposées 5 .

D'une manière plus générale, cette polémique s'inscrit dans le cadre de l'histoire déjà longue et cahotique des rapports culturels entre la ville de Nice et l'État: intervention de la ville dans la programmation du Centre dramatique national dirigé par Gabriel Monnet au début des années 1970; arrêt de l'aide de l'État aux quatre Maisons des jeunes et de la culture après que la ville, les ayant

3. Ainsi Kanal, Art Press, Galerie Magazine ou Beawx-Arts.

4. La Cote des arts, juillet-août 1990.

5. Voir à ce propos l'article de Geneviève Breerette dans Le Monde (18 juillet), la rubrique de Catherine Millet dans Art Press (octobre) et la critique de Gérard Monnier, Des Beaux-Arts aux arts plastiques, Besançon, La Manufacture, 1991, p. 317-318, qui reste marquée, nous semble-t-il, par la polémique: • Qui ne voit le rapport étonnant et choquant qui se produit ici entre les cuvres et le lieu ?... Ne fait pas un musée d'art contemporain qui veut . 
accusées d'être des « foyers de révolution ", eut partiellement cessé de les financer et eut contribué ainsi à les asphyxier; doublement de ces MJC par cinq Centres d'animation de culture et de loisirs de la ville de Nice (CACEL) et par des Centres de diffusion et d'action culturelle (CEDAC), tous dirigés par des proches de Jacques Médecin; contentieux à propos de la transformation de la Villa Arson en Centre national d'art contemporain (1982-1984), sous l'impulsion de Claude Mollard, Michel Butor et Henri Maccheroni; ou encore, sur un registre plus symbolique, l'absence remarquée de Jack Lang, en décembre 1989, lors de l'inauguration du nouveau Théâtre qui accueille la troupe du Théâtre de Nice dirigée par Jacques Weber.

Partie prenante de l'image moderne d'une cité, la * politique culturelle *, un des nouveaux symboles du volontarisme municipal, participe, au plan national comme à l'échelon local, d'une instrumentalisation de la culture. Et même si, dans ce domaine, les lois de décentralisation ne bouleversent pas la configuration juridique, la place désormais affirmée et légitimée des élus dans le paysage politique français permet un renforcement des politiques locales auquel le champ culturel n'échappe pas. La singularité du système politique niçois, la personnalisation du débat dans une conjoncture marquée par la montée du Front national et des réflexes xénophobes font de cette polémique un révélateur des liens entre la politique et la culture.

En outre, ce débat a eu quelques répercussions sur la vie culturelle niçoise. Ainsi, la Cinquième foire internationale de l'art contemporain, Art Jonction, se déroule, du 6 au 9 juillet, sans la présence des principales galeries parisiennes. Une des rares présentes, la galerie Enrico Navarra, au lieu de présenter des dessins russes contemporains, expose "des propos d'artistes contre le racisme *: entre autres, un tableau écriture de Ben, des panneaux de Louis Cane établissant un lien entre le Front national, Jacques Médecin et la démagogie, ou encore la main de . Touche pas à mon pote. peinte en jaune sur fond de graffiti par Klasen. De même, à la veille du festival de Musiques actuelles NiceCôte d'Azur (les MANCA), le compositeur italien Luciano Berio, hôte d'honneur, retirait ses deux "récréations": l':Omaggio à Joyce * et *Visage . De plus, le festival fut interdit de journalistes à sa conférence de presse parisienne et bien oublié par la critique spécialisée. Quant au public, il sera également au rendezvous: le 14 novembre 1990, le MAMAC fêtera son $100000^{e}$ visiteur et le 21 juin 1991 , après une première année de fonctionnement, il affichera un bilan très positif, avec près de 196000 visiteurs.

Dans sa livraison de juillet-août 1990, la revue Artension soulignait les ambiguités de cette polémique : “Cette sombre histoire du musée de Nice a quatre avantages: elle fait de la pub à Arman, elle en fait à Médecin, elle en fait pour le musée et elle fait vendre les journaux... Tout est donc pour le mieux dans le meilleur de l'immonde!. .

La vie culturelle à Nice ne s'est pas apaisée pour autant. En juillet 1991, les élus locaux du Front national protestèrent contre l'installation des sculptures de l'artiste américain, qui s'était exilé en Europe en 1971 pour protester contre la guerre du Vietnam, Mark Di Suvero. En février 1992, la présence de Jean-Marie Le Pen à la tête de liste du Front national dans les Alpes-Maritimes pour les élections régionales souleva également la réprobation de la "société culturelle. locale. À l'initiative de l'Association pour la démocratie à Nice et dans les AlpesMaritimes, une pétition, que Nice-Matin refusa de publier, rassembla plus de quatre cents noms de plasticiens (Arman, Ben, 
Venet, Ernest Pignon-Ernest, Sosno, Pagès, Dolla), d'écrivains (Maunoury, Bens, Michel Kahn, Raynal, Grisolia) et de metteurs en scène (Penchenat, Monnet, Martinotty). Mais surtout, Pierre Chaigneau, conservateur du MAMAC, et Jacques Weber, directeur du CDN, prirent, contrairement à 1990, clairement position contre les idées de Jean-Marie Le Pen dans le cadre de cette protestation. Le départ de Jacques Médecin, la recomposition rapide du paysage politique local facilitèrent, sans aucun doute, ces prises de posi- tion d'une * société culturelle " désormais unanime dans son action ${ }^{1}$.

1. Sur la perte de contrôle du système par le : maire-patron. voir Alain Garrigou, - Le boss, la machine et le scandale. La chute de la maison Médecin ., Politix, 17, 1992, p. 7-35.

Professeur d'bistoire, chargé d'enseignement à l'Université de Boungogne, Philippe Poirrier, achève une thèse sur les politiques culturelles de la ville de Dijon. 